\title{
INFLUÊNCIA DE AGENTES COLOIDISANTES NO PROCESSO DE PELOTIZAÇÃO DE MINÉRIO DE FERRO*
}

\author{
Sandra Lúcia de Moraes ${ }^{1}$ \\ José Renato Baptista de Lima² \\ João Batista Ferreira Neto ${ }^{3}$
}

\section{Resumo}

O presente trabalho visou contribuir para o melhor entendimento do mecanismo inerente ao uso de agente coloidisante/dispersante no processo de pelotização de concentrado de minério de ferro. Avaliou-se a influência dos agentes coloidisantes em comparação aos aglomerantes Peridur e bentonita. Para este fim, foram realizados ensaios de bancada visando a identificação de possíveis ações destes agentes no grau de dispersão das misturas, bem como a sua influência na liberação/aglomeração dos finos. Os resultados destes parâmetros foram confrontados com os resultados de caracterização das pelotas confeccionadas com os diferentes agentes pelos quais foi possível identificar que os dispersantes tem forte atuação sobre o conteúdo de finos do concentrado de minério de ferro. A ação principal se dá pela remoção das partículas ultrafinas que recobrem os grãos mais grosseiros presentes na amostra e as dispersam, no caso dos dispersantes, aumentando a disponibilidade do conteúdo de finos $<4 \mu \mathrm{m}$, principalmente os finos $<1 \mu \mathrm{m}$ (coloides). No caso do aglomerante orgânico, observa-se também essa ação, no entanto, os finos presentes são aglomerados na faixa entre 26 e $4 \mu \mathrm{m}$.

Palavras-chave: Pelotização; Minério de ferro; Aglomerante; Agente coloidisante.

\section{EFFECT OF COLLOIDAL AGENT IN THE PROCESS OF PELLETIZING OF IRON ORE}

\section{Abstract}

The objective of this study was to evaluate the influence of colloidal agents compared to binders, Peridur and bentonite, in the pelletizing process of iron ore concentrate aiming to contribute with the understanding the action of these agents in the mechanisms of agglomeration of the pellets. With this goal, bench tests were carried out to identify possible actions of binders/dispersant in the degree of dispersion of the mixtures, as well as its influence on the liberation/agglomeration of the fine particles. The results of these parameters were compared with the results of the characterization of the pellets prepared with different binders/dispersants. By this study, it was identified that the dispersant has a strong influence on the fine particle content on the iron ore concentrate. The main action takes place by the removal of ultrafine particles overlying coarser grains in the sample and the scatter, in the case of dispersants, increasing the availability of fine content $<4$ micrometers. In the case of the organic binder, this action is also observed; however, the fines are agglomerated in the range between 4 and 26 micrometers.

Keywords: Pelletizing; Binder; Dispersant; Colloidal agent.

1 Engenheira Química, Doutora, Pesquisadora, Centro de Tecnologia em Metalurgia e Materiais, Instituto de Pesquisas Tecnológicas do Estado de São Paulo, São Paulo, SP, Brasil.

2 Engenheiro de Minas, Professor Doutor, Departamento de Engenharia de Minas e de Petróleo, Escola Politécnica da Universidade de São Paulo, SP, Brasil.

3 Engenheiro Metalurgista, Doutor, Pesquisador, Centro de Tecnologia em Metalurgia e Materiais, Instituto de Pesquisas Tecnológicas do Estado de São Paulo, São Paulo, SP, Brasil. 


\section{INTRODUÇÃO}

O uso de aglomerantes na pelotização a quente de minério de ferro visa cumprir duas funções: (1) aumentar a resistência da pelota antes do aquecimento (resistência verde); (2) evitar o colapso das pelotas durante a "queima", quando a expulsão dos gases gerados pela vaporização de água tende a destruir a pelota.

A bentonita é o aglomerante mais empregado industrialmente, e seu mecanismo de ligação no processo de ganho de resistência da pelota já foi amplamente estudado e compreendido.

Várias tentativas de resolver os problemas da utilização de bentonita no processo de aglomeração de minério de ferro visam a utilização de aglomerantes orgânicos, que apresentam as vantagens de não inserirem contaminantes durante o processo, serem utilizados em menor quantidade e de poderem ser eliminados de forma substancial durante a queima das pelotas, produzindo basicamente dióxido de carbono e vapor de água [1].

Os dois principais tipos de aglomerantes orgânicos são os à base de celulose e de poliacrilamidas.

Um levantamento de estudos realizados por Moraes [2], referentes ao uso de aglomerantes orgânicos no processo de pelotização de minério de ferro, mostra que a ação do aglomerante está sempre associada à interação com algum sal inorgânico ou dispersantes de caráter ácido ou básico (entendidos no presente trabalho como agentes dispersantes/coloidisantes). A Tabela resume alguns desses exemplos, os quais tiveram a comprovação da ação destes agentes por meio da avaliação da resistência mecânica das pelotas.

Tabela 1 - Estudos de aglomerantes orgânicos no processo de pelotização de minério de ferro [2]

\begin{tabular}{|c|c|c|c|c|}
\hline \multirow[t]{2}{*}{ Estudo (Patente $N^{\circ}$ ) } & \multirow[t]{2}{*}{ Aglomerante } & \multirow[t]{2}{*}{ Agente coloidisante } & \multicolumn{2}{|c|}{$\begin{array}{l}\text { Resistência à } \\
\text { compressão (kg) }\end{array}$} \\
\hline & & & Verde & Seca \\
\hline $\begin{array}{l}\text { Allied Colloids } \\
\text { Limited (EU } 0225171 \\
\text { - 1987) }\end{array}$ & Copolímero de acrilamida & $\begin{array}{l}\text { - Acrilato de sódio } \\
\left.\text { ( } \mathrm{Na}_{3} \mathrm{Citrato}\right) \\
\text { - Soda } \mathrm{Na}_{2} \mathrm{CO}_{3}\end{array}$ & $0,4-0,71$ & $\begin{array}{l}2,07- \\
6,02\end{array}$ \\
\hline $\begin{array}{l}\text { Allied Colloids } \\
\text { Limited (US } \\
4.684 .549-1987)\end{array}$ & $\begin{array}{l}\text { Copolímero de acrilamida } \\
\text { / Carboximetil celulose }\end{array}$ & $\begin{array}{l}\text { - Acrilato de sódio } \\
\left.\text { ( } \mathrm{Na}_{3} \mathrm{Citrato}\right) \\
\text { - Soda } \mathrm{Na}_{2} \mathrm{CO}_{3}\end{array}$ & $0,4-0,71$ & $2,07-6,02$ \\
\hline $\begin{array}{l}\text { Aqualon Company } \\
\text { (US 4.863.512 - } \\
\text { 1989) }\end{array}$ & Carboximetil celulose & $\begin{array}{l}\text { Tripolifosfato de } \\
\text { sódio }\end{array}$ & $0,77-1,54$ & $4,76-7,85$ \\
\hline $\begin{array}{l}\text { Peridur® Nobel (US } \\
5.698 .007-1997)\end{array}$ & $\begin{array}{l}\text { Carboximetil celulose } \\
\left.\text { (Peridur }{ }^{\circledR}\right)\end{array}$ & $\begin{array}{l}\text { Fontes de íons } \\
\text { hidroxila }(\mathrm{NaOH})\end{array}$ & --- & $0,64-5,4$ \\
\hline $\begin{array}{l}\text { Peridur® Nobel (US } \\
6.071 .325-2000)\end{array}$ & $\begin{array}{l}\text { Carboximetil celulose } \\
\text { (Peridur } 囚 \text { ), } \\
\text { poliacrilatos, } \\
\text { poliacrilamidas, derivados } \\
\text { de celulose, goma guar, } \\
\text { amido, dextrina, pectinas }\end{array}$ & $\begin{array}{l}\text { Fontes de íons } \\
\text { hidroxila }(\mathrm{NaOH})\end{array}$ & --- & $0,68-3,22$ \\
\hline $\begin{array}{l}\text { Clariant S.A. Brasil } \\
\text { (EP } 2548978 \text { A1 - } \\
\text { 2013) }\end{array}$ & $\begin{array}{l}\text { Homopolímero de ácido } \\
\text { acrílico, ácido metacrílico, } \\
\text { acrilamida }\end{array}$ & Calcário/esmectita & $\begin{array}{l}1,24-1,61 / \\
1,44-2,61\end{array}$ & $2,9-6,59$ \\
\hline
\end{tabular}

Das patentes verificadas, apenas a EP 2548978 A1 (2013) cita a realização de um teste para verificação do grau de dispersão obtido com os dispersantes avaliados. No entanto, os resultados obtidos não são apresentados naquele documento. 
Alguns artigos científicos abordando o emprego de aglomerantes para pelotização de minério de ferro são apresentados na Tabela.

Tabela 2 - Artigos científicos sobre aglomerantes orgânicos para pelotização de minério de ferro [2]

\begin{tabular}{|c|c|c|c|c|}
\hline \multirow{2}{*}{$\begin{array}{l}\text { Minério de } \\
\text { ferro }\end{array}$} & \multirow{2}{*}{ Aglomerante } & \multicolumn{2}{|c|}{ Caracterização } & \multirow{2}{*}{ Referência } \\
\hline & & Aglomerantes & Pelotas & \\
\hline $\begin{array}{l}\text { Hematita } \\
\text { (CVRD) }\end{array}$ & $\begin{array}{l}\text { Peridur }{ }^{\circledR} \mathrm{e} \\
\text { bentonita }\end{array}$ & Não & $\begin{array}{l}\text { Quedas, compressão } \\
\text { (verde, seca) }\end{array}$ & $\begin{array}{l}\text { SOUZA et al. } \\
(1984)\end{array}$ \\
\hline Magnetita & Peridur $®$ & Não & $\begin{array}{l}\text { Quedas, compressão } \\
\text { (verde, seca) }\end{array}$ & $\begin{array}{l}\text { KATER e } \\
\text { STEEGHS } \\
(1984)\end{array}$ \\
\hline $\begin{array}{l}\text { Taconito } \\
\text { (Minesota, } \\
\text { USA) }\end{array}$ & $\begin{array}{l}\mathrm{CMC}\left(\mathrm{Na}_{2} \mathrm{CO}_{3},\right. \\
\mathrm{NaCl}), \text { Peridur }{ }^{2} \\
\text { bentonita }\end{array}$ & $\begin{array}{l}\text { Grau de substituição, } \\
\text { viscosidade }\end{array}$ & $\begin{array}{l}\text { Quedas, compressão } \\
\text { (verde, seca e } \\
\text { queimada), } \\
\text { propriedades } \\
\text { químicas e } \\
\text { metalúrgicas }\end{array}$ & $\begin{array}{l}\text { GOETZMAN et. } \\
\text { al. (1988) }\end{array}$ \\
\hline Magnetita & $\begin{array}{l}\text { Aglomerantes } \\
\text { orgânicos e } \\
\text { bentonita }\end{array}$ & $\begin{array}{l}\text { PWA (plate water } \\
\text { absorption test) } \\
\text { Viscosidade, tensão }\end{array}$ & $\begin{array}{l}\text { Quedas, compressão } \\
\text { (verde, seca e } \\
\text { queimada) }\end{array}$ & $\begin{array}{l}\text { HAAS et al. } \\
\text { (1989) }\end{array}$ \\
\hline $\begin{array}{l}\text { Magnetita } \\
\text { (Carol Plant, } \\
\text { Canadá) }\end{array}$ & $\begin{array}{l}\text { Aglomerantes } \\
\text { orgânicos (inclusive } \\
\text { Peridur } 囚 \text { ) e } \\
\text { bentonita }\end{array}$ & Não & $\begin{array}{l}\text { Quedas, compressão } \\
\text { (verde, seca e } \\
\text { queimada), } \\
\text { propriedades } \\
\text { metalúrgicas }\end{array}$ & $\begin{array}{l}\text { MARTINOVIC et } \\
\text { al. (1989) }\end{array}$ \\
\hline $\begin{array}{l}\text { Hematita } \\
\text { (CVRD) }\end{array}$ & CMC, TPP & Não & $\begin{array}{l}\text { Quedas, compressão } \\
\text { (verde, seca) }\end{array}$ & LIMA (1991) \\
\hline $\begin{array}{l}\text { Hematita } \\
\text { (Ferteco) }\end{array}$ & $\begin{array}{l}\text { CMC, TPP, } \\
\text { aglomerantes } \\
\text { orgânicos }\end{array}$ & Não & $\begin{array}{l}\text { Quedas, compressão } \\
\text { (verde, seca) }\end{array}$ & $\begin{array}{l}\text { CASSOLA e } \\
\text { CHAVES (1998) }\end{array}$ \\
\hline $\begin{array}{l}\text { Pellet feed } \\
\text { (China) }\end{array}$ & $\begin{array}{l}\text { S-1 (Tipo de amido } \\
\text { modificado) e Funa } \\
\text { (substância rica em } \\
\text { ácido húmico obtida } \\
\text { do linhito por meio } \\
\text { de extração por } \\
\text { solução cáustica) }\end{array}$ & $\begin{array}{l}\text { Design molecular, } \\
\text { molhabilidade, } \\
\text { potencial de } \\
\text { ionização, afinidade } \\
\text { de elétrons, energia } \\
\text { de ligação }\end{array}$ & $\begin{array}{l}\text { Quedas, compressão } \\
\text { (verde, seca), } \\
\text { choque térmico }(820 \\
\left.\text { e } 780^{\circ} \mathrm{C}\right)\end{array}$ & QIU et al. (2003) \\
\hline $\begin{array}{l}\text { Pellet feed } \\
\text { (Rússia) }\end{array}$ & $\begin{array}{l}\text { (Floform } 1049 \mathrm{~V}) \\
\text { poliacrilamida }\end{array}$ & Não & $\begin{array}{l}\text { Propriedades físicas, } \\
\text { químicas e } \\
\text { metalúrgicas }\end{array}$ & $\begin{array}{l}\text { CHIZHIKOVA e } \\
\text { VAINSCHTEIN } \\
(2003)\end{array}$ \\
\hline $\begin{array}{l}\text { Concentrado } \\
\text { de minério de } \\
\text { ferro }\end{array}$ & $\begin{array}{l}\text { HS (Substâncias } \\
\text { húmicas extraídas } \\
\text { de linhito) }\end{array}$ & $\begin{array}{l}\text { Viscosidade em } \\
\text { função do pH e da } \\
\text { temperatura, } \\
\text { adsorção }\end{array}$ & $\begin{array}{l}\text { Quedas, compressão } \\
\text { (verde, seca), } \\
\text { choque térmico }(550 \\
\text { e } 600^{\circ} \mathrm{C} \text { ) }\end{array}$ & $\begin{array}{l}\text { ZHANG et al. } \\
(2011)\end{array}$ \\
\hline $\begin{array}{l}\text { Pellet feed } \\
\text { (Baiama } \\
\text { Plant - } \\
\text { China) } \\
\end{array}$ & $\begin{array}{l}\text { MHA (ácido fúlvico } \\
\text { e ácido húmico) }\end{array}$ & $\begin{array}{l}\text { Viscosidade, potencial } \\
\text { zeta, pH, cinética de } \\
\text { adsorção }\end{array}$ & $\begin{array}{l}\text { Quedas, compressão } \\
\text { (verde, seca), } \\
\text { queima }\end{array}$ & $\begin{array}{l}\text { HAN et al. } \\
\text { (2012) }\end{array}$ \\
\hline
\end{tabular}

A grande maioria dos artigos destacados na Tabela faz referência ao desempenho do aglomerante considerando os aspectos de resistência à compressão e a quedas, comparativamente ao desempenho da bentonita. Uma linha de investigação um tanto quanto diferenciada dos demais foi abordada nos trabalhos realizados por Goetzman et. al. [3]; Haas et al. [4] e Qiu et al.[5], os quais procuram associar algum resultado de caracterização dos aglomerantes avaliados com as características de resistência mecânica das pelotas. 
Observa-se pelas patentes e artigos analisados a inexistência de uma fundamentação teórica da ação destes materiais nos mecanismos formação e, consequentemente, sua influência nas propriedades mecânicas das pelotas produzidas.

No presente trabalho vislumbrou-se o melhor entendimento dos mecanismos da ação de agentes dispersantes/coloidisantes na aglomeração das pelotas de concentrado de minério de ferro.

O objetivo geral desse trabalho é contribuir para o melhor entendimento do mecanismo inerente ao uso de agente coloidisante na aglomeração do material particulado no processo de pelotização de concentrado de minério de ferro.

\section{MATERIAIS E MÉTODOS}

Os trabalhos experimentais foram divididos em duas etapas, sendo que a primeira compreendeu a avaliação do efeito do uso de dispersantes nas propriedades reológicas e coloidais de partículas ultrafinas de minério de ferro. Nesta fase foi realizado um levantamento do estado da arte neste tema visando a obtenção de parâmetros para comprovação prática da ação dos dispersantes no processo de pelotização. Os parâmetros selecionados a partir da literatura foram: viscosidade, potencial zeta, grau de dispersão e conteúdo de finos.

Para a realização dos trabalhos foi utilizado um concentrado de minério de ferro brasileiro tipo pellet feed, oriundo da indústria, denominados Minério $A$, cujo $P_{80}$, fração na qual oitenta por cento do material é passante, é 54,4 $\mu \mathrm{m}$.

Em uma segunda etapa, foram realizados ensaios de pelotização e caracterização das pelotas obtidas, quais sejam: umidade, resistência a quedas, resistência à compressão das pelotas verdes e secas. Os resultados obtidos nesta etapa foram confrontados com cada parâmetro obtido na primeira etapa, visando o entendimento da influência dos mesmos nas características de resistência mecânica das pelotas.

Os aglomerantes avaliados foram bentonita nacional e o orgânico Peridur. Avaliouse também um tipo de CMC grau técnico, ou seja, não tratada para fins aglomerantes. Os dispersantes utilizados são produtos de uso comercial. A Tabela 3 apresenta os produtos avaliados e as respectivas dosagens aplicadas.

Tabela 3 - Aglomerantes/agentes coloidisantes utilizados nos trabalhos

\begin{tabular}{|c|c|c|c|}
\hline Aglomerante/agente coloidisante & Fórmula química & Fabricante & Adição (\%) \\
\hline Bentonita Nacional & - & ---- & 0,5 \\
\hline Aglomerante orgânico Peridur® 330 & $\begin{array}{l}\text { Sodium } \\
\text { CarboxymethylCellulose }\end{array}$ & Akzo Nobel & 0,04 \\
\hline $\begin{array}{l}\text { Carboximetilcelulose (CMC) pura - Grau } \\
\text { técnico (DS - 0,46) }\end{array}$ & $\left.{ }^{R O} L_{-} O^{O R}\right)^{R} R=\mathrm{Hor}$ & $\begin{array}{l}\text { Denver Especialidades } \\
\text { Químicas }\end{array}$ & 0,04 \\
\hline Carbonato de sódio P.A. & $\mathrm{Na}_{2} \mathrm{CO}_{3}$ & $\begin{array}{l}\text { Labsynth Produtos } \\
\text { para Laboratórios Ltda }\end{array}$ & 0,02 \\
\hline Dispersol® A99 & $\begin{array}{l}\text { Homopolímero de ácido } \\
\text { acrílico neutralizado com } \\
\text { hidróxido de cálcio }\end{array}$ & Clariant S.A. & $0,02 / 0,15$ \\
\hline Hidróxido de sódio (escamas) & $\mathrm{NaOH}$ & Impex & 0,02 \\
\hline Tripolifosfato de sódio (TPP) grau técnico & $\mathrm{Na}_{5} \mathrm{P}_{3} \mathrm{O}_{10}$ & Anidrol & 0,15 \\
\hline
\end{tabular}

Os ensaios de pelotização foram realizados em escala de bancada com alíquotas de concentrado de minério de ferro de cerca de $5 \mathrm{~kg}$ (base úmida), utilizando-se o disco de pelotização marca Lurgi. A mistura dos componentes a pelotizar foi sempre 
realizada em misturador intensivo de laboratório marca Eirich Indústria Ltda., modelo R02E.

\section{RESULTADOS E DISCUSSÃO}

\subsection{Avaliação de Dispersantes/Aglomerantes nas Propriedades Reológicas dos Concentrados de Minério de Ferro}

Esta fase dos trabalhos teve como objetivo obter parâmetros que caracterizassem a ação dos aglomerantes/dispersantes quando em solução com o concentrado de minério de ferro. Levantou-se como suposição a aplicação de parâmetros reológicos como forma de avaliar os efeitos da aplicação de aglomerantes que atuam como dispersantes ou vice-versa. Um levantamento do estado da técnica apresentado em Moraes et al. [6], permitiu identificar parâmetros para a proposição de um método de avaliação do efeito de aglomerantes antes da etapa de pelotização propriamente dita, para minério de ferro. Assim, as misturas de concentrado minério de ferro e aglomerantes/dispersante foram avaliadas quanto à: conteúdo de finos (análise granulométrica); viscosidade; potencial zeta e grau de dispersão.

Embora o trabalho tenha contemplado a avaliação dos parâmetros destacados acima, neste documento serão discutidos apenas os resultados obtidos relacionados a influência dos dispersantes/aglomerantes no conteúdo de finos e no grau de dispersão do concentrado de minério de ferro estudado.

\subsubsection{Avaliação do conteúdo de finos}

A variação do conteúdo de finos pela ação dos aglomerantes/dispersantes foi avaliada por meio de análise granulométrica por difração a laser das misturas em relação ao conteúdo apresentado pelo minério tal qual. As Figuras 1, 2 e 3 ilustram a distribuição da diferença do conteúdo de finos, tomando o ponto zero como a linha do minério de ferro tal qual, apresentada em termos de porcentagem retida simples. Os resultados negativos significam que houve redução de material naquela faixa, enquanto que o valor positivo indica que houve aumento.

Os dados da Figura 1 referem-se a avaliação dos dispersantes, adicionados em baixa dosagem $(0,02 \%)$, em relação a atuação do aglomerante orgânico Peridur.

Conforme apresentado na Figura 1, o aglomerante orgânico Peridur tem ação intensa sobre as partículas de minério de ferro, a qual é notada desde a fração 80 $\mu \mathrm{m}$. Há uma redução da fração entre 80 e $26 \mu \mathrm{m}$ de cerca de $22 \%$ e um consequente incremento de partículas na fração entre 26 e $4 \mu \mathrm{m}$, da ordem de $25 \%$. A adição de $\mathrm{NaOH}$ juntamente com o aglomerante orgânico não altera esta condição, no entanto, reduz a ação nessas faixas para cerca de 18 e 14\%, respectivamente. Em ambos os casos há uma diminuição de cerca de $3 \%$ do conteúdo de finos na fração $<4 \mu \mathrm{m}$.

Comportamento similar à ação do aglomerante orgânico é notado quando o $\mathrm{NaOH} e$ o dispersante Dispersol são aplicados com dosagem de 0,02\%, porém a intensidade é menor. Entretanto, estes dois agentes têm ação inclusive na fração $<4,0 \mu \mathrm{m}$, aumentado em cerca de $10 \%$, em relação ao minério de ferro tal qual, o conteúdo de finos nesta fração. 


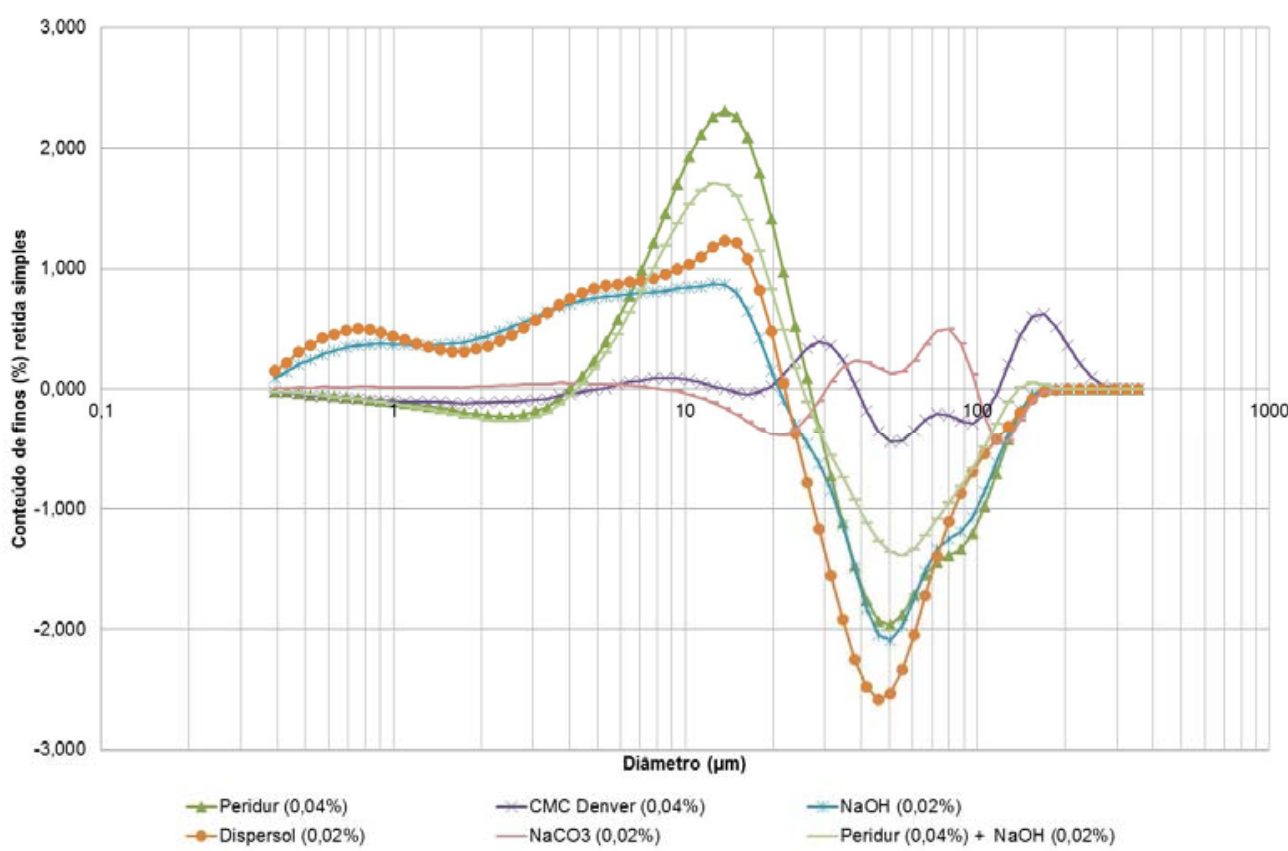

Figura 1. Diferença do percentual de finos em relação ao minério tal qual, empregando aglomerante orgânico e dispersantes em baixas dosagens.

O carbonato de sódio e a CMC pura não exercem influência no conteúdo de finos do minério de ferro. Cabe ressaltar que, intencionalmente, a CMC utilizada nesse trabalho é um produto grau técnico, ou seja, uma CMC não purificada, uma vez que o objetivo desse estudo era avaliar as implicações decorrentes do emprego de agentes dispersantes/coloidisantes de origem comercial no processo de aglomeração. O desempenho da CMC como aglomerante para pelotização está associado aos seguintes parâmetros físicos-químicos: grau de substituição (DS), grau de polimerização (DP), peso molecular, viscosidade, ativação, dentre outros. Estes parâmetros são definidos conforme o minério a ser aglomerado.

A Figura 2 apresenta as distribuições da diferença de conteúdo de finos obtidas incluindo o uso dos dispersantes em dosagem mais alta $(0,15 \%)$ em relação ao aglomerante orgânico. Conforme se observa na Figura 2, a ação dos dispersantes em dosagens mais altas $(0,15 \%)$ é mais acentuada sobre o conteúdo de finos. 0 dispersante Dispersol é o que exerce a maior influência nas frações entre 80 e 26 $\mu \mathrm{m}$, reduzindo em cerca de $45 \%$ o conteúdo de material nessa faixa e aumentando em cerca de $20 \%$ o conteúdo de finos menores que $4 \mu \mathrm{m}$.

A Figura 3 apresenta os resultados comparados ao da bentonita. A bentonita tem ação semelhante à ação dos dispersantes e diferenciada da ação do aglomerante orgânico Peridur. Nota-se uma diminuição do conteúdo de finos na faixa de 80 a 26 $\mu \mathrm{m}$ e um aumento na fração entre 26 e $4 \mu \mathrm{m}$ e na fração menor que $4 \mu \mathrm{m}$. No entanto, os valores encontrados são menores que quando do uso de dispersantes. 


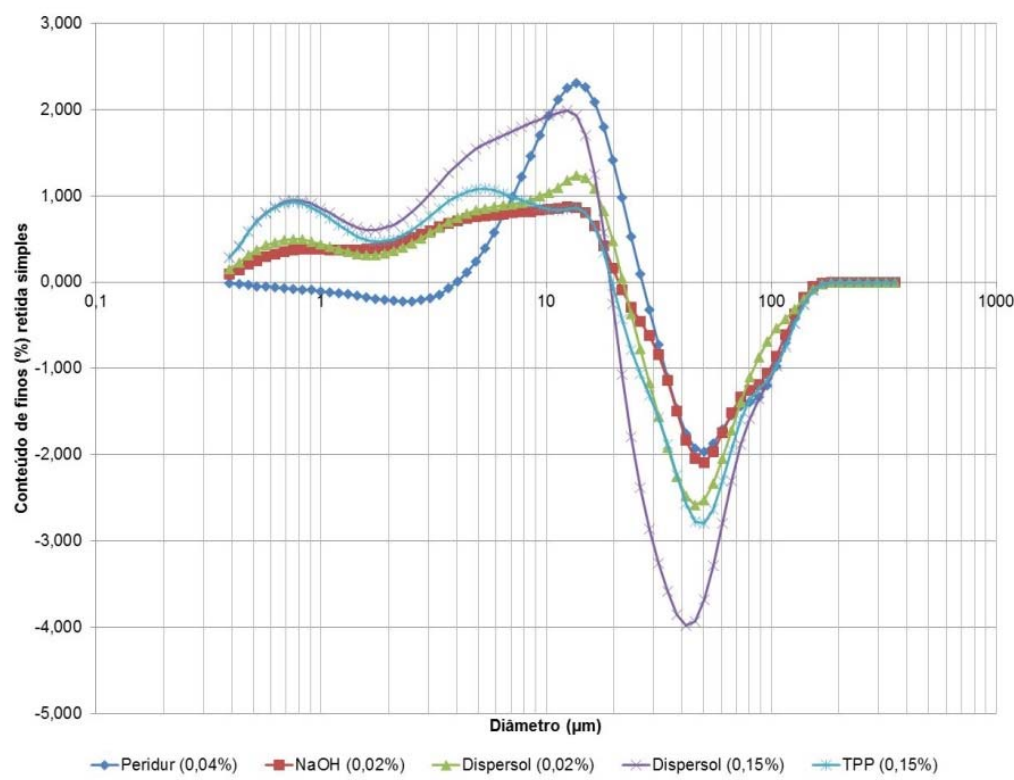

Figura 1Diferença do conteúdo de finos em relação ao minério tal qual: aglomerante orgânico e dispersantes alta dosagem.

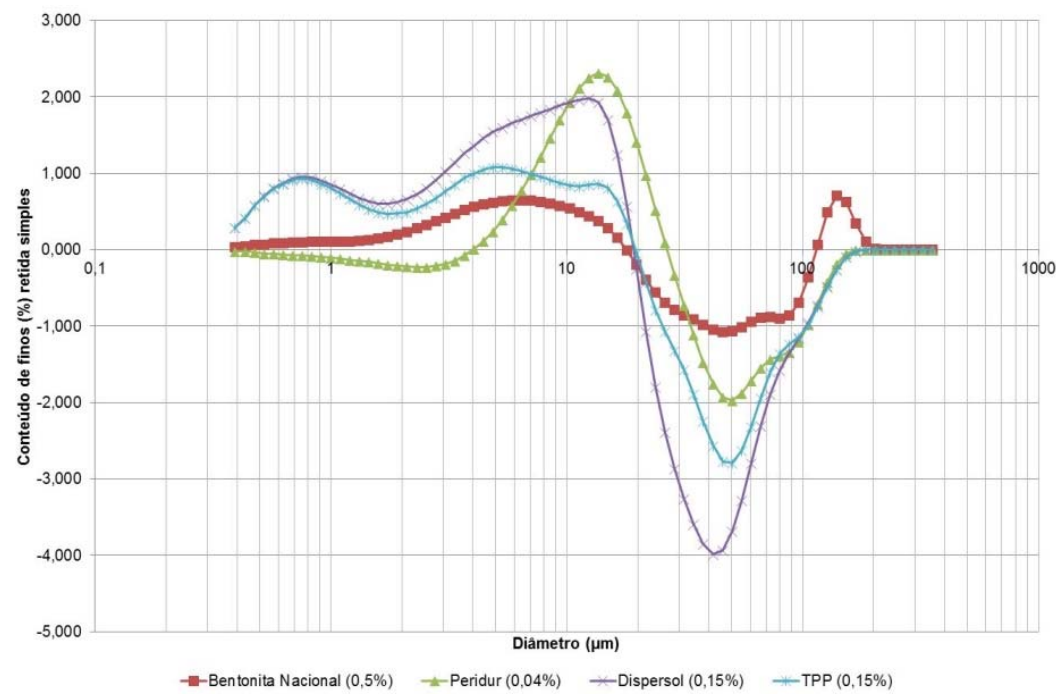

Figura 3. Diferença do conteúdo de finos em relação ao minério tal qual, empregando aglomerante orgânico, dispersantes baixa dosagem e bentonita.

\subsubsection{Avaliação do grau de dispersão}

Os ensaios de dispersão foram realizados em escala de bancada utilizando $2,5 \mathrm{~g}$ de mistura (minério de ferro+aglomerante). Após agitação manual por 15 segundos e repouso por 7 minutos para sedimentação do material em proveta, o material sobrenadante e o sedimentado foram retirados separadamente e secos. O grau de dispersão foi obtido a partir da equação $D=100^{*} \mathrm{Ms} / \mathrm{Mr}$, onde $\mathrm{Ms}$ é a massa do sobrenadante após secagem e Mr é massa do sedimentado após secagem.

Os resultados dos ensaios para determinação do grau de dispersão das misturas estão apresentados na Tabela 4. O maior grau de dispersão foi obtido pelo uso de TPP $(8,57 \%)$, seguido do Dispersol $(7 \%)$ nas dosagens indicadas nesta tabela.

O aglomerante orgânico Peridur reduz o grau de dispersão do minério de ferro, que é da ordem de $3 \%$, para $2 \%$; sendo que quando se aplica o aglomerante Peridur com o $\mathrm{NaOH}$ este valor cai para cerca de 1\%. Observa-se uma correlação do grau 
de dispersão com a quantidade de finos $<4 \mu \mathrm{m}$ liberados na mistura, ou seja, quanto maior o conteúdo de finos nesta faixa, maior o grau de dispersão.

\begin{tabular}{ccc}
\multicolumn{3}{l}{ Tabela 4 - Grau de dispersão das amostras } \\
\hline Ensaio & Composição & Grau de dispersão (D) \\
\hline $\mathbf{0}$ & Minério A (100\%) & 3,33 \\
$\mathbf{2}$ & Bentonita nacional $(0,5 \%)$ & 4,53 \\
$\mathbf{3}$ & Peridur (0,04\%) & 2,02 \\
$\mathbf{9}$ & Peridur (0,04\%) + NaOH $(0,02 \%)$ & 1,21 \\
$\mathbf{4}$ & $\mathrm{CMC} \mathrm{Denver} \mathrm{(0,04 \% )}$ & 5,31 \\
$\mathbf{8}$ & $\mathrm{Na}_{2} \mathrm{CO}_{3}(0,02 \%)$ & 4,88 \\
$\mathbf{5}$ & $\mathrm{NaOH}(0,02 \%)$ & 5,31 \\
$\mathbf{6}$ & Dispersol $(0,02 \%)$ & 6,97 \\
$\mathbf{7}$ & Dispersol $(0,15 \%)$ & 7,38 \\
$\mathbf{1 0}$ & TPP $(0,15 \%)$ & 8,57
\end{tabular}

\subsection{Avaliação do Uso de Dispersantes nos Mecanismos de Aglomeração de Pelotas de Minério de Ferro}

A Tabela 5 apresenta os resultados obtidos nos ensaios de pelotização de concentrado de minério de ferro quanto à umidade, resistência a quedas e resistência à compressão em função do aglomerante/dispersante utilizado.

Tabela 5 - Resultados dos ensaios de pelotização em função do aglomerante/dispersante utilizado nas concentrações indicadas

\begin{tabular}{clcccc}
\hline Ensaio & \multicolumn{1}{c}{ Composição } & Umidade & Quedas & $\begin{array}{c}\text { Resistência compressão } \\
\text { (kgf/pel) } \mathbf{\pm} \mathbf{P}_{\mathbf{9 5}}\end{array}$ \\
\hline & & $(\%)$ & $(45 \mathrm{~cm}) \pm \mathrm{P}_{95}$ & verde & seca \\
$\mathbf{0}$ & Minério ferro $(100 \%)$ & 10,96 & $3,1 \pm 0,35$ & $0,79 \pm 0,12$ & $1,04 \pm 0,08$ \\
$\mathbf{2}$ & Bentonita nacional $(0,5 \%)$ & 11,01 & $4,5 \pm 1,35$ & $1,15 \pm 0,07$ & $4,06 \pm 0,36$ \\
$\mathbf{3}$ & Peridur $(0,04 \%)$ & 11,47 & $5,4 \pm 0,73$ & $1,01 \pm 0,10$ & $1,59 \pm 0,12$ \\
$\mathbf{9}$ & Peridur $(0,04 \%)+\mathrm{NaOH}(0,02 \%)$ & 10,95 & $6,3 \pm 1,65$ & $1,58 \pm 0,11$ & $6,57 \pm 0,67$ \\
$\mathbf{5}$ & NaOH $(0,02 \%)$ & 10,48 & $3,3 \pm 0,51$ & $1,01 \pm 0,20$ & $1,32 \pm 0,19$ \\
$\mathbf{4}$ & CMC Denver $(0,04 \%)$ & 9,49 & $3,8 \pm 0,79$ & $1,12 \pm 0,12$ & $1,18 \pm 0,09$ \\
$\mathbf{6}$ & Dispersol $(0,02 \%)$ & 8,72 & $2,9 \pm 0,62$ & $1,20 \pm 0,08$ & $1,2 \pm 0,16$ \\
$\mathbf{7}$ & Dispersol $(0,15 \%)$ & 9,54 & $2,5 \pm 0,33$ & $1,51 \pm 0,14$ & $5,43 \pm 0,17$ \\
$\mathbf{8}$ & Na ${ }_{2} \mathrm{CO}(0,02 \%)$ & 10,21 & $3,5 \pm 0,33$ & $1,30 \pm 0,12$ & $1,18 \pm 0,19$ \\
$\mathbf{1 0}$ & $\mathrm{TPP}(0,15)$ & 10,11 & $3,1 \pm 0,63$ & $1,55 \pm 0,38$ & $6,81 \pm 0,72$ \\
\hline
\end{tabular}

Em relação ao aglomerante Peridur e aos dispersantes utilizados, percebe-se que há correlação da resistência à compressão a verde com o conteúdo de finos $<26 \mu \mathrm{m}$. Isto é, a resistência à compressão a verde aumenta com o aumento do conteúdo de finos $<26 \mu \mathrm{m}$ presentes na mistura. No caso do aglomerante Peridur, o aumento da resistência a verde pode ser atribuída à ação de retirada das partículas finas da superfície das partículas mais grosseiras e ao processo de aglomeração dessas partículas na faixa entre 26 e $4 \mu \mathrm{m}$ o que causa maior plasticidade da pelota. Observa-se um incremento de resistência quando do uso do aglomerante Peridur $+\mathrm{NaOH}$ em cerca de $56 \%$. 


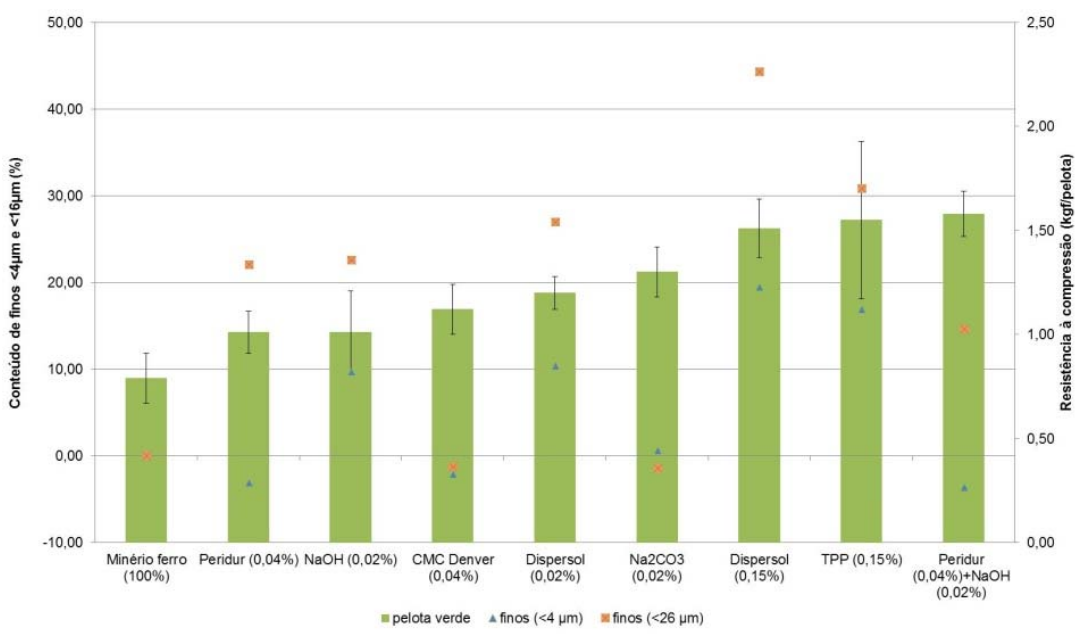

Figura 4. Efeito do percentual de finos $<4 \mu \mathrm{m} \mathrm{e}<26 \mu \mathrm{m}$ sobre a resistência à compressão de pelotas verdes obtidas com a presença dos aglomerantes orgânicos CMC e Peridur e dos agentes dispersantes.

Os resultados de resistência à compressão das pelotas secas associados ao conteúdo de finos proporcionado pelos aglomerantes/dispersantes utilizados nesse trabalho estão ilustrados na Figura 5. Como se pode observar, tanto o $\mathrm{NaOH}$ como Dispersol, no nível de dosagem de $0,02 \%$, embora atuem no aumento do conteúdo de finos $<26 \mu \mathrm{m}$ e $<4 \mu \mathrm{m}$, não causaram um ganho de resistência da pelota seca no nível de concentração empregado. No entanto, em níveis de dosagem mais altos, o valor de resistência à compressão supera o mínimo estipulado pelas pelotizadoras que é de $3,5 \mathrm{kgf} /$ pelota.

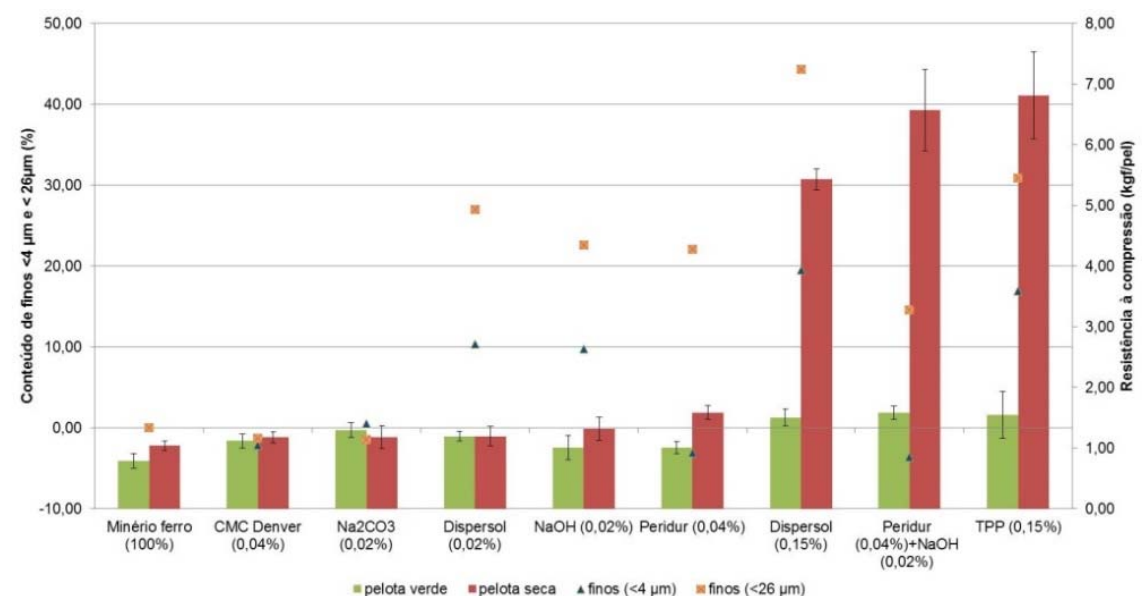

Figura 5. Correlação entre a resistência à compressão das pelotas secas e o percentual de finos, decorrente do emprego de diferentes aglomerantes/dispersantes nas concentrações indicadas.

Conforme apresentado anteriormente, os dispersantes utilizados na dosagem de $0,02 \%$ tem atuação similar a do aglomerante orgânico, porém agem também sobre os finos menores que $1 \mu \mathrm{m}$ (coloides). Este efeito é mais intenso em dosagens mais altas. No caso do emprego do Dispersol, na concentração de $0,15 \%$, há um incremento de $91 \%$ do conteúdo de finos $<1 \mu \mathrm{m}$ em relação à de $0,02 \%$. Além disso, o aumento da resistência à compressão pode ser atribuído ao incremento do conteúdo de material coloidal, o qual amplia a ação das forças inter-partículas, principalmente a de van der Waals, a qual é particularmente significante quando as partículas são coloidais $(<1 \mu \mathrm{m})$ [7]. Esta mesma explicação se estende também a atuação do TPP $(0,15 \%)$ como aglomerante. 
Embora o aglomerante Peridur tenha proporcionado a estabilidade da pelota verde, o mesmo não foi capaz de garantir a ganho de resistência à compressão da pelota na etapa de secagem para o concentrado de minério de ferro avaliado. No entanto, o emprego do aglomerante Peridur em conjunto com o $\mathrm{NaOH}$ proporcionou um incremento significativo da resistência a compressão da pelota seca $(6,58$ kgf/pelota), a qual é cerca de 1,58 vezes superior ao mínimo estipulado.

Conforme já abordado, o aglomerante Peridur tem uma forte atuação sobre a superfície das partículas grosseiras diminuindo a quantidade de finos presentes na faixa de 80 a $26 \mu \mathrm{m}$ e aumentando a quantidade de finos entre 26 e $4 \mu \mathrm{m}$, condição tal que não é significativamente alterada pela adição de $\mathrm{NaOH}$. Neste caso, não é possível atribuir o fenômeno de remoção/aglomeração dos finos ao ganho da resistência da pelota seca ao uso do $\mathrm{NaOH}$ em conjunto com o aglomerante Peridur.

\section{CONCLUSÃO}

Pelo presente trabalho foi possível comprovar que os agentes coloidisantes atuam sobre os finos presentes no minério de ferro. $O$ agente coloidisante adicionado puro favorece a aglomeração, uma vez que disponibiliza os finos coloidais presentes na amostra para o meio, sendo sua atuação mais intensa em função da quantidade de finos disponíveis no sistema. Ao passo que, ao ser adicionado como parte integrante do aglomerante orgânico, sua atuação se dará por meio de dispersão, seguida de floculação por parte de outros componentes do aglomerante, a qual agrupará as partículas coloidais na faixa entre 26 e $4 \mu \mathrm{m}$.

\section{Agradecimentos}

Os autores agradecem a FAPESP - Fundação de Amparo à Pesquisa do Estado de São Paulo pelo suporte financeiro a este projeto, no âmbito da Chamada Faps_Vale.

\section{REFERÊNCIAS}

1 KOGEL, J.E.; TRIVERDI, N.C.; BARKER, J.M.; KRUKOWSKI, S. Industrial minerals \& rocks: commodities, markets and uses. $7^{\text {th }}$. Littleton: SME, 2006. $1548 \mathrm{p}$.

2 MORAES, S.L. Contribuição ao entendimento do efeito de agente coloidisante no processo de pelotização de concentrado de minério de ferro. 2014. 138 p. Tese (Doutorado) - Escola Politécnica, Universidade São Paulo, São Paulo, 2014.

3 GOETZMAN, H.E.; BLEIFUSS, R.L.; ENGESSER, J. Investigation of carboxymethylcellulose binders for taconite pelletizing. Littleton: SME, 1988. $44 \mathrm{p}$. (Preprint - Society of Mining Engineers of AIME). Apresentado originalmente em Conferencia da SME, realizado em Luxembourg, 2-3 Oct. 1986.

4 QIU, G.; JIANG, T.; LI, H.; WANG, D. Functions and molecular structure of organic binders for iron ore pelletization. Colloids and Surfaces A: Physicochemical and Engineering Aspects, v. 224, p. 11-22, 2003.

5 HAAS, L.A.; ALDINGER, J.A.; ZAHL, R.K. Effectiveness of organic binders for iron ore pelletization. Pittsburgh, PA: U.S. Department of the Interior. Bureau of Mines, 1989. (Report of Investigations, RI 9230).

6 MORAES, S. L.; LIMA, J. R. B. ; FERREIRA NETO, J. B. . Influence of dispersants on the rheological and colloidal properties of iron ore ultrafine particles and their effect on the pelletizing process-A review. Journal of Materials Research and Technology, v. 2, p. 386391, 2013.

7 HARTLEY, P.A.; PARFITT, G.D.; POLLACK, L.B. The role of the Van Der Waals force in the agglomeration of powders containing submicron particles. Powder Technology, v. 42, p. 35-46, 1985. 